\title{
Evolutionary history of black grouse major histocompatibility complex class IIB genes revealed through single locus sequence-based genotyping
}

Tanja Strand ${ }^{1,2}$, Biao Wang ${ }^{1}$, Yvonne Meyer-Lucht ${ }^{1}$ and Jacob Höglund ${ }^{1 *}$

\begin{abstract}
Background: Gene duplications are frequently observed in the Major Histocompatibility Complex (MHC) of many species, and as a consequence loci belonging to the same MHC class are often too similar to tell apart. In birds, single locus genotyping of MHC genes has proven difficult due to concerted evolution homogenizing sequences at different loci. But studies on evolutionary history, mode of selection and heterozygosity correlations on the MHC cannot be performed before it is possible to analyse duplicated genes separately. In this study we investigate the architecture and evolution of the MHC class IIB genes in black grouse. We developed a sequence-based genotyping method for separate amplification of the two black grouse MHC class IIB genes BLB1 and BLB2. Based on this approach we are able to study differences in structure and selection between the two genes in black grouse and relate these results to the chicken MHC structure and organization.

Results: Sequences were obtained from 12 individuals and separated into alleles using the software PHASE. We compared nucleotide diversity measures and employed selection tests for BLB1 and BLB2 to explore their modes of selection. Both BLB1 and BLB2 are transcribed and display classic characteristics of balancing selection as predicted for expressed MHC class IIB genes. We found evidence for both intra- and interlocus recombination or gene conversion, as well as indication for positive but differential selection at both loci. Moreover, the two loci appear to be linked. Phylogenetic analyses revealed orthology of the black grouse MHC class IIB genes to the respective BLB loci in chicken.

Conclusions: The results indicate that the duplication of the BLB gene occurred before the species divergence into black grouse, chicken and pheasant. Further, we conclude that BLB1 and BLB2 in black grouse are subjected to homogenizing concerted evolution due to interlocus genetic exchange after species divergence. The loci are in linkage disequilibrium, which is in line with the theory of tightly coevolving genes within the MHC under the minimal essential MHC hypothesis. Our results support the conclusion that MHC form and function in birds derived from studies on the domesticated chicken are not artefacts of the domestication process.
\end{abstract}

Keywords: Balancing selection, Concerted evolution, Early duplication, BLB1, BLB2, Galliformes, 3'UTR, Tetrao tetrix

\footnotetext{
* Correspondence: jacob.hoglund@ebc.uu.se

'Population Biology and Conservation Biology, Department of Ecology and Genetics, Evolutionary Biology Center, Uppsala University, Norbyvägen 18D, SE-752 36, Uppsala, Sweden

Full list of author information is available at the end of the article
} 


\section{Background}

The duplication of genes is a common process in the evolution and adaptation of most organisms [1]. Understanding the molecular mechanisms that shaped duplicated genes is essential to study the evolutionary history and selection processes acting on complex gene families in a larger context. A very prominent example of a multigene family with frequent genetic and genomic duplications is the Major Histocompatibility Complex (MHC), which holds a key role in the vertebrate adaptive immune response in pathogen recognition and defense [2]. Thereby, MHC genes are linked to individual fitness and the outcome of infections (reviewed in e.g. [3]). The genes of the MHC are known to be the most polymorphic and among the best studied functional genes in vertebrates [4]. In humans, for instance, close to a thousand different alleles are known from the MHC HLADRB1 locus [5]. The extreme polymorphism commonly found at MHC genes is believed to be maintained by means of balancing selection, driven by selection through diverse pathogens and leading to maintenance of allelic variation within populations [6-8]. Balancing selection acts on variation at the MHC by means of heterozygote advantage (dominant as well as overdominant selection), rareallele advantage (negative frequency-dependent selection) and/or selection that fluctuates over space and time [3]. Gene conversion and recombination [9], trans-species evolution [10], MHC-dependent sexual selection (reviewed in [4]) and selection against hitchhiking recessive deleterious mutations [11] have also been shown to play a role in shaping $\mathrm{MHC}$ diversity.

Duplications of MHC loci are frequently observed, and as a consequence loci belonging to the same MHC class are, in many species, too similar to distinguish by their allelic sequences [12-16]. However, studies on the evolutionary history, the mode of selection and heterozygosity correlations on the MHC cannot be performed before it is possible to analyse duplicated loci separately [4]. Single locus amplification of polymorphic MHC genes in birds has been a goal for evolutionary biologists for over a decade [12,17], but has not been achieved until recently. Very few bird species studied so far have only a single MHC class IIB locus (i.e. the green-rumped parrotlet [18], penguins [19] and kestrels [20]). Among the numerous birds with duplicated MHC class IIB loci, single locus amplification has so far only been reported in red jungle fowl/domestic chicken (Gallus gallus) [21,22], captive turkey (Meleagris gallapavo) [23] and Barn owl (Tyto alba) [24].

The MHC shows pronounced differences in genomic organization and in number of MHC loci between vertebrate lineages [25], especially when mammalian and non-mammalian species are compared [26,27]. Different hypotheses have emerged for how the complex MHC family has evolved. The prevailing consensus is that $\mathrm{MHC}$ evolution is characterized by repeated gene duplication (birth) and gene loss (death), whereby the loci evolve under a birth-and-death model [2,28,29]. Phylogenetic relationships can reveal whether gene duplication arose post speciation or pre speciation. Post speciation duplication leads to a pattern where paralogous $\mathrm{MHC}$ loci within a species are more closely related than orthologous MHC loci between species [30,31]. Pre speciation duplication, in contrast, took place in an ancestral species and results in orthologous MHC loci from different species being more closely related than paralogous MHC loci within species. However, these patterns can likewise be generated by different processes. A situation identical to what to be expected under a post speciation duplication event can be caused by concerted evolution acting on early duplicated genes [26,32]. Under concerted evolution, gene fragments are frequently exchanged between paralogous loci, and thereby homogenized (interlocus genetic exchange by recombination or gene conversion $[9,33])$. However, if convergent selection acts on either different loci within a species or between different species, those loci will functionally converge and mask the actual evolutionary relationship $[32,34]$. These different processes are not mutually exclusive, which adds a further level of difficulty to the interpretation of evolutionary patterns at the MHC.

The domestic chicken (Gallus gallus domesticus) is the major avian $\mathrm{MHC}$ model species with a history of more than 60 years of MHC studies [35]. The core of MHC in the chicken is called the classical $\mathrm{MHC}$ or the $\mathrm{BF} / \mathrm{BL}$ region which, among others, consists of MHC class I and class II genes, TAP genes, Tapasin and one MHC class IV (BG) gene. This gives a total of 19 genes in $92 \mathrm{~kb}$ [36] situated on a single chromosome compared to the human MHC region which comprises 128 expressed genes as well as pseudo genes on a stretch of $3600 \mathrm{~kb}$ on several chromosomes [37]. The chicken MHC also appears more compact than what has been observed in the passerine bird which is best known, the zebra finch (Taeniopygia guttata), in which the MHC region is more complex and found on at least four chromosomes [38]. Because of the compact and small structure of the chicken MHC compared to the mammalian MHC, and the presence of few expressed genes, it has been named "a minimal essential MHC". In addition, the chicken $\mathrm{MHC}$ is arranged differently than the mammalian MHC [36]. MHC class I and class II genes are tightly linked $[31,39]$, and TAP genes has been demonstrated to coevolve with MHC class I (BF) genes [31]. It has also been suggested that other MHC genes may co-evolve [30,40]. Based on experiments, recombination within BF/BL in the chicken is rarely observed [41] but sequencing showed evidence of gene conversion and recombination in 
shaping the chicken MHC [39]. Furthermore, examinations of $\mathrm{BF} / \mathrm{BL}$ in pedigreed families in the closely related galliforms turkey and Japanese quail (Coturnix japonica) have revealed direct evidence of gene conversion and recombination $[42,43]$.

In the chicken, both MHC class I (BF) and MHC class IIB (BLB) comprise two loci but only one locus in each class is highly expressed. While the BLB2 locus is considered to be dominantly expressed [44,45], the BLB1 locus has been suggested not be involved in peptide binding and to be neutral to selection $[30,46]$. Nevertheless, interlocus genetic exchange has been observed between BLB1 and BLB2 in a farmed population of pheasant (Phasianus colchicus) and in the domestic chicken, i.e. a case of concerted evolution [47]. In this study we test whether BLB1 and BLB2 evolved independently or in a concerted way in a wild close relative to both the pheasant and the chicken: the black grouse (Tetrao tetrix). Disentangling the evolutionary history of BLB1 and BLB2 in black grouse will add valuable information about how these genes evolved in the chicken and related species. To date, it is largely unknown if the uniquely compact MHC in the chicken [36] is a result of the long domestication process or was present before domestication.

Domestic chicken and black grouse belong to the same avian family (Phasianidae; Galliformes) and both possess two MHC class II B (BLB) loci that surround the Tapasin gene (Figure 1) [44,48]. Although the MHC class II B region is well studied in the domestic chicken, no primers could be developed for independent amplification of either MHC class II B minor (BLB1) or MHC class II B major (BLB2) [49]. The chicken BLB1 and BLB2 are nearly identical in sequence and can only be amplified separately with PCR primers anchored outside the BLB genes along with a nested non-locus-specific PCR $[22,44]$.

In this study, we present an approach to genotype the two MHC class II B loci separately in a wild galliform, the black grouse. The procedure comprises a long-range anchored PCR method followed by nested PCR reactions and sequence-based genotyping. Based on this approach we first aim to characterize and contrast MHC class II B diversity and mode of selection at the BLB1 and BLB2 loci in the black grouse. Second, we explore whether the two loci underlie concerted evolution or evolve independently. And third, we relate and compare the results to the MHC class II B structure and organization in the chicken.

\section{Methods}

\section{Samples}

We studied locus-specific MHC class IIB variation in twelve black grouse individuals, eleven of which were genotyped non locus-specific in our previous studies $[15,50]$. We included one more individual (JHGO 213) and genomic DNA was extracted from tissue with a salt extraction protocol [51]. In addition, we used sequence data obtained in a fosmid sequencing study on the same individual JHGO 213 [48] [GenBank JQ028669]. Sequences from other species used in the data analysis derived from GenBank, accession numbers are given in the respective figure legends.

\section{Locus-specific amplification of BLB1 and BLB2}

In order to amplify the two black grouse MHC class IIB genes BLB1 and BLB2 separately, we applied a longrange anchored PCR method combined with nested non locus-specific PCRs. For each BLB locus, we anchored one of the PCR primers outside of the respective BLB

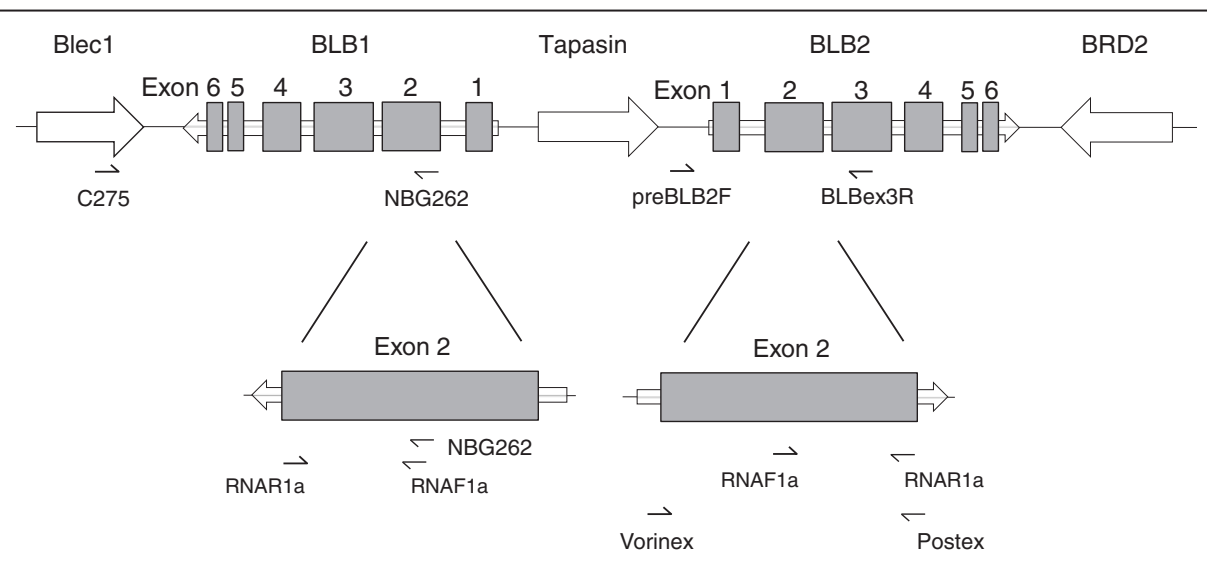

Figure 1 Schematic figure of the genomic focus region of the MHC. Genes are illustrated by white arrows indicating their orientation, with the names above the drawing. The exon-intron structure is given for BLB1 and BLB2 to show the target region and primer positions. The positions and amplification directions of all primers used in this study are indicated by small one-headed arrows. Below the upper part of the drawing, the primers for the long-range PCR amplifications of BLB1 and BLB2 (C275, NBG262 and preBLB2F, BLBex3R) are given. Further down, the target region Exon 2 is enlarged, with the positions of the nested PCR primers (BLB1: RNAR1a, RNAF1a, NBG262; BLB2: Vorinex, RNAF1a, RNAR1a, Postex) given below. Note that BLB1 and BLB2 are orientated in opposite directions. 
gene for a locus-specific long-range PCR (Figure 1). In a second step, these long-range PCR fragments were used as template in nested PCRs with non locus-specific primers to amplify parts of the BLB exon 2 .

We selectively amplified a long fragment of the BLB1 gene with a modification of primers designed for the chicken BLB minor [44]. We used the forward primer $\mathrm{C} 275$ located in the Blec1 gene adjacent to BLB1 gene, and the reverse primer NBG262, slightly modified from C262 situated within BLB1 exon 2 (Figure 1) (see Additional file 1 for primer sequences). These primers yielded a $1932 \mathrm{bp}$ PCR product. All attempts to design primers amplifying the complete BLB1 exon 2 based on chicken genome sequences or on black grouse MHC fosmid data failed. In the $20 \mu \mathrm{l}$ BLB1 long-range reaction, approximately $100 \mathrm{ng}$ DNA, $0.3 \mu \mathrm{M}$ of each primer C275 and NBG262, $0.8 \mu \mathrm{M}$ dNTP, $0.6 \mu$ DMSO, 1x Phusion GC buffer and $1 \mathrm{U}$ Phusion High-Fidelity DNA polymerase (Finnzymes, Espoo, Finland) were used. The PCR programme was initiated with $40 \mathrm{~s}$ at $98^{\circ} \mathrm{C}$, followed by 35 cycles of $10 \mathrm{~s}$ at $98^{\circ} \mathrm{C}, 20 \mathrm{~s}$ at $62^{\circ} \mathrm{C}$ and $68 \mathrm{~s}$ at $72^{\circ} \mathrm{C}$ and ended with $6 \mathrm{~min}$ at $72^{\circ} \mathrm{C}$.

Based on the black grouse MHC fosmid data [GenBank JQ028669] [48] we designed primers for a selective amplification of the BLB2 gene. We imported the BLB2 sequence and flanking regions to the NCBI web based primer design program Primer BLAST [52] and marked areas that were conserved between the black grouse BLB2, the chicken $B L B$ major and the turkey BLB2 (Meleagris gallopavo), but were different from BLB1. The resulting primer pair forward preBLB2F and reverse BLBex3R yielded a 1359 bp PCR product ranging from the intragenic region between the Tapasin gene and BLB2 to the BLB2 exon 3 (Figure 1) (see Additional file 1 for primer sequences). For amplification of the BLB2 we used approximately $100 \mathrm{ng}$ DNA, $0.3 \mu \mathrm{M}$ of each primer preBLB2F and BLBex3R, $0.3 \mu \mathrm{M}$ KAPA dNTP, 1x KAPA GC buffer and 0.5 U KAPA HiFi DNA polymerase (KAPA BIOSYSTEMS, Boston, United States) in a $25 \mu$ l reaction. The PCR programme was initiated with $5 \mathrm{~min}$ at $98^{\circ} \mathrm{C}$, followed by 35 cycles of $20 \mathrm{~s}$ at $98^{\circ} \mathrm{C}, 15 \mathrm{~s}$ at $62^{\circ} \mathrm{C}$ and $90 \mathrm{~s}$ at $72^{\circ} \mathrm{C}$ and ended with a final extension for $5 \mathrm{~min}$ at $72^{\circ} \mathrm{C}$.

Long-range PCR products for BLB1 and BLB2 revealed each one strong single band on a 1.5\% agarose gel. A sterile toothpick was inserted into each band, taking up a small amount of the PCR product, and transferred into $50 \mu \mathrm{ldd} \mathrm{H}_{2} \mathrm{O}$. This solution was stored overnight at $-20^{\circ} \mathrm{C}$ before using it in the nested PCRs as template as described in the following.

\section{Nested PCR to amplify exon 2 and sequencing}

The primers RNAF1a and RNAR1a [15] (Additional file 1) were used in a nested PCR to amplify $125 \mathrm{bp}$ on the BLB1 and BLB2 long-range PCR products as template. $1 \mu \mathrm{l}$ of the template solution, $0.48 \mu \mathrm{M}$ of each primer, $0.6 \mathrm{mM}$ of dNTP, $3 \mathrm{mM} \mathrm{MgCl} 2,1 \mathrm{x}$ buffer and $0.75 \mathrm{U}$ BioTaq DNA polymerase (DNA Technology, Aarhus, Denmark) were used in a $25 \mu \mathrm{l}$ PCR reaction. The PCR programme was initiated at $94^{\circ} \mathrm{C}$ for $5 \mathrm{~min}$, followed by 30 cycles at $94^{\circ} \mathrm{C}$ for $1 \mathrm{~min}$ and $30 \mathrm{~s}$ at both $64.9^{\circ} \mathrm{C}$ and $72^{\circ} \mathrm{C}$, and ended for $10 \mathrm{~min}$ at $72^{\circ} \mathrm{C}$. We also performed another nested PCR amplifying a longer part of the exon 2 on the BLB2 longrange PCR product only. A second primer pair, Vorinex2 and Postex2, developed for the closely related willow grouse (Lagopus lagopus), was used to amplify 251 bp of exon 2 (see Additional file 1). $1 \mu$ l of the template solution, $0.4 \mu \mathrm{M}$ of each primer, $0.48 \mathrm{mM}$ of $\mathrm{dNTP}, 1 \mathrm{x} \mathrm{KCl}$ buffer and $1 \mathrm{U}$ Taq DNA polymerase (Fermentas, St. Leon-Rot, Germany) were used in a $25 \mu \mathrm{l}$ PCR reaction. The programme was initiated at $94^{\circ} \mathrm{C}$ for $5 \mathrm{~min}$, followed by 30 cycles at $94^{\circ} \mathrm{C}$ for $1 \mathrm{~min}$ and $30 \mathrm{~s}$ at both $66^{\circ} \mathrm{C}$ and $72^{\circ} \mathrm{C}$ before a final extension for $10 \mathrm{~min}$ at $72^{\circ} \mathrm{C}$. Using the primer pair Vorinex2/Postex2 a longer portion of BLB2 exon 2 sequences were amplified that were not known from before, so every new 251 bp sequence was subjected to verifying PCR runs (both BLB2 long-range PCR and nested PCR) and sequencing. For BLB1, the $251 \mathrm{bp}$ nested fragment could not be amplified due to the position of the nested primer Vorinex2 outside of the BLB1 long-range PCR product.

The nested PCR products were cleaned with an ExoSap reaction (Exonuclease I-Shrimp Alkaline Phospate, Fermentas, St. Leon-Rot, Germany). Cycle sequencing reactions were performed in both directions and the sequencing products were subjected to a post-reaction clean-up (GE Healthcare, Uppsala, Sweden), following the protocols of the manufacturer. All direct sequencing was performed both forward and reverse on a MegaBACE 1000 DNA analysing system (GE Healthcare, Uppsala, Sweden).

\section{Confirmation of MHC alleles}

PCR artefacts are a large issue in MHC studies and cautions to reduce the formation of artificial alleles are important to address [53]. To decrease the probability of PCR artefacts we only regarded alleles present in two independent PCR reactions as confirmed [54]. For the long-range PCR reactions for BLB1 and BLB2 we used different enzymes (Phusion High-Fidelity DNA polymerase and KAPA HiFi DNA), which could produce different levels of PCR artefacts. We were able to compare the sequences derived by the long-range PCR presented here, with previously cloned sequences using the non locus-specific primers RNAF1a and RNAR1a directly on genomic DNA [15,50]. Between 16 and 26 clones were sequenced per individual in the previous studies. We found that enzymes in the long-range PCRs of BLB1 and 
BLB2 worked equivalently well in amplifying alleles corresponding to those previously found (Table 1).

\section{Data analysis \\ Identification of sequences and analysis of sequence variation}

CodonCode Aligner version 3.7.1 was used for sequence editing and performing ambiguity codes for heterozygous positions. Allelic phase for heterozygote sequences was determined by computational inference with the PHASE haplotype reconstruction algorithm in DnaSP version 5.10.05 [55]. The PHASE algorithm has been proven reliable for determining the allelic phase in a number of previous MHC studies (e.g. [56,57]). The parameters used were 1000 iterations, thinning 10, 100 burn in, and a recombination model. For the haplotype reconstruction of the 125 bp fragment we included sequences that were previously verified by cloning. Sequence variation statistics were calculated in DnaSP version 5.10.05 [55] including the number of segregating sites, nucleotide diversity $(\pi)$ and average number of nucleotide differences (theta $k$ ).

\section{Recombination, gene conversion and linkage}

The power of any method for the detection of recombination or gene conversion strongly depends on the number of sequences included, the recombination rate and the number of sites differentiating the recombinant sequences [58,59]. Therefore it is suggested to apply an array of different methods to analyse recombination. We used a set of seven methods to detect recombination signals and putative recombinant sequences. The minimum number of recombination events $\left(\mathrm{R}_{\mathrm{m}}\right)$ according to the four-gamete test by Hudson \& Kaplan [60] was calculated in DnaSP version 5.10.05 [55]. This method tests for overall evidence of recombination in the alignment. We used the RDP3 package [61] to apply the methods RDP [62], Maxchi [63], Chimaera [64] and GENECONV [65]. These methods are designed to detect recombination breakpoint locations; the first three use a dynamic sliding window, whereas GENECONV searches for unusual long regions of identity between sequences. Moreover, we used the method GARD [66] implemented in Datamonkey [67], which likewise identifies recombination breakpoints, searching all possible partitions in a probabilistic way. Recombination rate $\rho$ and mutation rate $\theta$ were calculated using LDhat recombination rate scan [68].

We tested for linkage between the BLB1 and the BLB2 locus applying a likelihood ratio test of linkage disequilibrium implemented in Arlequin 3.5 [69]. In this test, the likelihood of the sample evaluated under the hypothesis of no association between loci (linkage equilibrium) is compared to the likelihood of the sample when association is allowed (linkage disequilibrium).

\section{Analysis of selection}

Positive selection is the spread to fixation of an allele that increases the fitness of individuals [70]. Initially, a locus undergoing balancing selection seems to be subjected to positive selection, but then experiences negative selection on alleles that became too frequent (under frequency dependent selection). Testing for long-term balancing selection in genetic regions therefore often includes testing for positive selection,

Table 1 Allele designations for the twelve black grouse individuals included in this study

\begin{tabular}{|c|c|c|c|c|c|}
\hline Individual & $\mathrm{BLB}_{125}$ & $\mathrm{BLB2}_{125}$ & BLB2 $_{251}$ & $\begin{array}{c}\text { Summary } \\
\text { BLB1 \& BLB2 }\end{array}$ & $\begin{array}{l}\text { BLB cloning } \\
\text { Non-specific* }\end{array}$ \\
\hline D248 & 06 & 07,12 & 07,12 & $06,07,12$ & $04,06,07,12$ \\
\hline D249 & 06,11 & 09,10 & 09,10 & $06,09,10,11$ & 06,11 \\
\hline D320 & 03,06 & 01,07 & 01A, 07 & $01 \mathrm{~A}, 03,06,07$ & $01,06,07$ \\
\hline D375 & 05 & 01 & 01A, 01B & 01A, 01B, 05 & 01,05 \\
\hline D476 & 05 & 01, 09 & 01A, 09 & $01 \mathrm{~A}, 05,09$ & $01,05,09$ \\
\hline D870 & 03,04 & 01,02 & $01 \mathrm{~A}, 02$ & $01 \mathrm{~A}, 02,03,04$ & (cDNA) $01,02,03,04$ \\
\hline D115804 & 04,14 & 01,04 & 01C, 04B & 01C, 04, 04B, 14 & 01,04 \\
\hline $\mathrm{H} 070$ & 05,06 & 01,09 & 01B, 09 & 01B, 05, 06, 09 & $01,05,06$ \\
\hline $\mathrm{H} 071$ & 04,05 & 01,02 & 01B, 02 & $01 B, 02,04,05$ & $01,02,04,05$ \\
\hline $\mathrm{H} 369$ & 05 & 01,02 & $01 \mathrm{~A}, 02$ & $01 \mathrm{~A}, 02,05$ & $01,02,05$ \\
\hline $\mathrm{H} 393$ & 05,06 & 01,04 & 01B, 04B & 01B, 04B, 05, 06 & $01,04,05,06$ \\
\hline $\mathrm{JHGO} 213$ & 04 & 01,02 & $01 \mathrm{~A}, 02$ & $01 \mathrm{~A}, 02,04$ & no \\
\hline $\mathrm{H}_{\text {obs. }}$ & 0.58 & 0.92 & 1 & & \\
\hline $\mathrm{N}$ of alleles & 6 & 7 & 9 & minimum 14 & 11 \\
\hline
\end{tabular}

*Previous non locus-specific cloning from [15,50]. 
as inferred from the ratio of non-synonymous/synonymous $(d N / d S$ or $\omega)$ substitution rates, for instance [71]. Therefore, the outcome of methods can reflect a positive selection process, although in our context, testing for positive selection within a species is translated into balancing selection.

Tajima's D, Fu and Li's D and F tests were analysed in DnaSP version 5.10.05 [55] and Fisher exact tests were employed to test for differences from neutrality. In addition to the averaged Tajima's D, we performed sliding window Tajima's D with a window size of $11 \mathrm{bp}$ and a step size of $1 \mathrm{bp}$ for the $125 \mathrm{bp}$ fragments of the black grouse BLB1 and BLB2. A negative Tajima's D (as Fu and Li's D, Fu and Li's F) is a sign of negative selection or population expansion and a positive value is a sign of positive/balancing selection or a population bottleneck. Averaged synonymous $(d S)$ and non-synonymous $(d N)$ substitutions per synonymous and non-synonymous site were calculated in MEGA 5.05 [72]. The Nei-Gojobori method with Jukes Cantor corrections and 5000 bootstrap replicates was used to calculate the overall average of $d N / d S(\omega)$ for all sites, the peptide binding region (PBR) and non-PBR according to [73]. Deviations from neutrality $(d N=d S)$ were assessed by a Z-test. Differences in $d N$ and $d S$ between PBR and non-PBR were tested for significance using Mann-Whitney $U$-tests.

A different approach to detect molecular evidence of positive selection is to calculate $\omega$ per codon using the maximum likelihood method CODEML implemented in PAML version 4.6 [74]. The programme estimates heterogeneous $\omega$ among sites applying different models of codon evolution. We compared M2a (allowing $\omega$ to vary between $\omega<1$ (conserved), $\omega=0$ (neutral) and $\omega>1$ (positive)) with M1a ( $\omega$ allowed to vary between $\omega<1$ and $\omega=0)$ and M8 ( $\omega$ can vary in beta distribution of 0 and 1 including $\omega>1$ ) with M7 ( $\omega$ allowed to vary in a beta distribution of 0 and 1) using a log-likelihood test. Significant positive selection was inferred if twice the difference in log-likelihood values between the two models was greater than the $x^{2}$ critical value for the given degrees of freedom. The Bayes empirical Bayes (BEB) approach was used to identify significantly positively selected codon sites. Tree files used in PAML analyses were generated using a maximum likelihood approach in PhyML3.0 [75], under the F81 model of nucleotide substitution and estimated gamma shape parameter. Models of nucleotide substitution and the distribution of rate variation across nucleotide sites (gamma) were estimated in jModelTest 0.1.1 [76]. The best molecular evolution models were selected by the Aikaike information criterion (AIC) (see Additional file 2).

\section{Reconstruction of phylogenetic relationships}

We constructed phylogenetic networks based on the BLB exon 2 sequences and on the $3^{\text {rd }}$ codon positions of exon 2 only to illustrate the phylogenetic relationship among the black grouse BLB1 and BLB2 alleles and possible orthology to the chicken BLB1 and BLB2. The networks were built with the software SplitsTree4, using the neighbour net method [77]. Phylogenetic trees based on exon 3 sequences and on 3' untranslated regions (UTRs) were constructed using the Neighbour-joining method with bootstrapping (1000 replicates) implemented in MEGA 5.0 [72]. For BLB orthology between the black grouse and the chicken, we particularly looked at the 3'UTRs, since orthology between the pheasant and the chicken BLB1 and BLB2 has previously been demonstrated with the $3^{\prime}$ UTRs [47].

\section{Results}

\section{Locus-specific genotyping of black grouse BLB}

We successfully amplified locus-specific long-range PCR products for both BLB1 and BLB2 in all twelve black grouse individuals. In subsequent nested PCR reactions, we amplified and sequenced a $125 \mathrm{bp}$ PBR-rich fragment of the exon 2 for both loci, and almost the whole exon 2 for the BLB2 locus (251 out of $270 \mathrm{bp}$ ). Positions and sequences of the different primers are given in Figure 1 and Additional file 1. All heterozygous sequences could be assigned to haplotypes using the software PHASE implemented in DnaSP [55]. At the BLB1 locus we detected 6 unique $125 \mathrm{bp}$ alleles (BLB1 125 , Table 1, Figure 2) and at the BLB2 locus 7 unique 125 bp alleles (BLB2 ${ }_{125}$ ) and 9 unique $251 \mathrm{bp}$ alleles $\left(B L B 2_{251}\right)$. None of these sequences contained an indel or a stop codon, we thus assume that they are functional.

At the $\mathrm{BLB1}_{125}$ locus seven out of twelve individuals were heterozygous, whereas at the BLB2 all but one individual $\left(B L B 2_{125}\right)$, respective all individuals $\left(B L B 2_{251}\right)$ were heterozygous (Table 1 ). For allele BLB2 ${ }_{125^{-0}} 01$ the $251 \mathrm{bp}$ fragment revealed additional variation towards the $5^{\prime}$-end of exon 2 . This resulted in a subdivision into $\mathrm{BLB} 2_{251}-01 \mathrm{~A}, \mathrm{BLB} 2_{251}-01 \mathrm{~B}$ and $\mathrm{BLB} 2_{251}-01 \mathrm{C}$, each differing by one amino acid exchange (Figure 2). We found one $125 \mathrm{bp}$-allele that occurred at both BLB loci, namely $\mathrm{BLB}_{125}-04$. There is indication, however, that $\mathrm{BLB}_{125}-04$ differs at the $5^{\prime}$-end of exon 2 between the two loci [see Additional file $3, \mathrm{BLB}_{251}-04 \mathrm{~B}$ and $\mathrm{BLB}_{202}-04-\mathrm{cDNA}$, the latter from our previous study [15].

All individuals displayed unique $\mathrm{MHC}$ class II B genotypes. Combining the data for BLB1 and BLB2, 12 individuals had a minimum of 14 unique BLB alleles and each individual carried between three and four alleles. In comparison, 11 out of the 12 individuals previously analysed by non locus-specific BLB cloning [15,50] revealed 11 unique BLB alleles and individuals carried between two and four alleles (Table 1). This was a minor underestimation of both the number of unique alleles and the number of alleles per individual due to the 


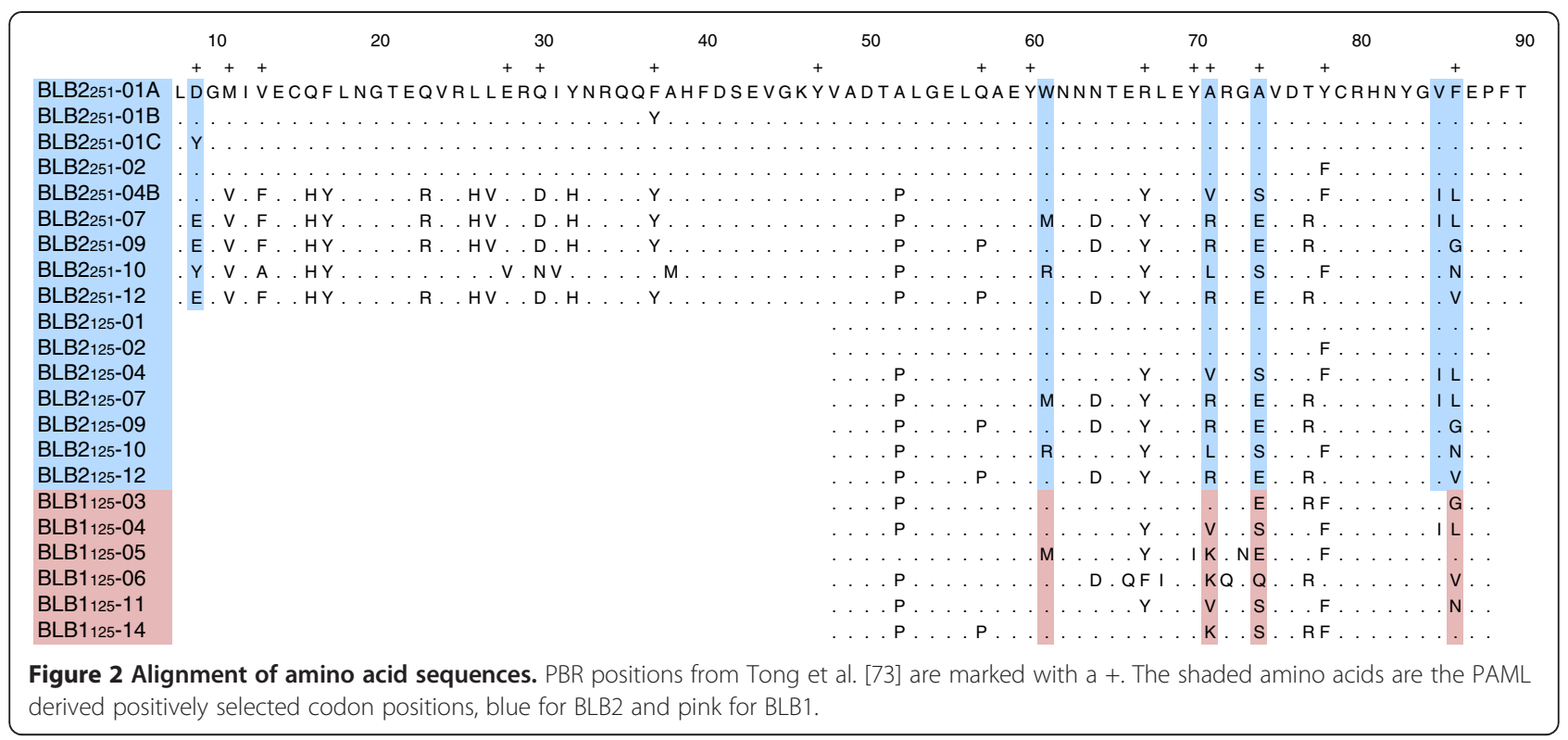

applied method compared to the here presented more accurate single locus amplifications.

There is evidence that both BLB loci are expressed in the black grouse. In an earlier study we found four BLB sequences in one individual by non locus-specific cloning based on cDNA (D870, Table 1, [15]). Our current study reveals that two of these sequences stem from BLB1 and the other two from BLB2.

\section{Sequence analyses: gene diversity, recombination and linkage}

Although the locus $B L B 1_{125}$ showed a lower number of unique alleles than $B L B 2_{125}$, it revealed more segregating sites, a higher number of average pairwise differences theta $k$ and a higher nucleotide diversity $\pi$ (Table 2). As expected, the longer fragment $B L B 2_{251}$ revealed more alleles, more segregating sites, a higher theta $k$ but a lower $\pi$ than the shorter $B L B 2_{125}$. For $B L B 1_{125}$, 7 out of 8 positions involved in antigen binding [73] were polymorphic, whereas 6 out of 8 and 12 out of 15 positions were variable for BLB2 ${ }_{125}$ and BLB2 251 , respectively (Figure 2).

We used a set of different methods to detect recombination signals and putative recombinant sequences, and found evidence for recombination in each of the fragments $\left(\mathrm{BLB}_{125}, \mathrm{BLB} 2_{125}, \mathrm{BLB} 2_{251}, \mathrm{BLB} 1 \& 2_{125}\right)$, at least with the four-gamete test and the method Maxchi (Table 3). These recombination signals lead to a number of recombinant sequences including both intra- and interlocus recombination or gene conversion (Table 4). Considering both loci together $\left(B L B 1 \& 2_{125}\right)$ the estimated population recombination rate $\rho$ was 11.42 , and the mutation rate $\theta$ was 9.27 , leading to a recombinationmutation ratio of 1.23 . A $\rho / \theta$ ratio of $>1$ is an indication for recombination being more prevalent in the dataset than point mutations [78]. For the loci separately, however, the estimated mutation rates were much higher than $\rho$, leading to $\rho / \theta$ ratios $<1$. The results of the different tests combined strongly suggest that recombination or gene conversion has occurred in the black grouse $\mathrm{MHC}$ class II.

We observed significant linkage disequilibrium between the BLB1 and BLB2 (likelihood-ratio-test, LnLikelihood LD: -46.087, LnLikelihood LE: -62.120, exact $\mathrm{p}=0.0239$ ), indicating that the two loci are linked.

\section{Analyses of selection}

Positive values for Tajima's D, Fu \& Li's D and Fu \& Li's $\mathrm{F}$ were detected for $\mathrm{BLB}_{125}$ and $\mathrm{BLB}_{251}$ (Table 2),

\section{Table 2 Gene diversity measures}

\begin{tabular}{|c|c|c|c|c|c|c|c|}
\hline Locus & A & $S$ & Theta $k$ & $\pi$ & Tajima's D & Fu \& Li's D & Fu \& Li's F \\
\hline $\mathrm{BLB1}_{125}$ & 6 & 34 & 15.87 & 0.13 & -0.74 & -0.52 & -0.62 \\
\hline$B L B 2_{125}$ & 7 & 23 & 12.29 & 0.09 & 0.43 & 0.91 & 0.88 \\
\hline$B L B 2_{251}$ & 9 & 43 & 20.42 & 0.08 & 0.56 & 0.53 & 0.60 \\
\hline $\mathrm{BLB} 1 \& 2_{125}$ & 12 & 35 & 13.79 & 0.11 & -0.25 & -0.01 & -0.08 \\
\hline
\end{tabular}

Number of unique alleles (A), number of segregating sites (S), average pairwise differences (theta $k$ ), nucleotide diversity ( $\pi$ ) and neutrality test summaries for the different fragments of BLB1 and BLB2. All neutrality tests were non-significant (all $p>0.10$ ). 
Table 3 Number of recombination events calculated by different methods

\begin{tabular}{lcccccccccc}
\hline Locus & No. of sequences & $\mathbf{R}_{\mathbf{M}}$ & RDP & GENECONV & Maxchi & Chimera & GARD & $\boldsymbol{\rho}$ & $\boldsymbol{\theta}$ & $\boldsymbol{\rho} / \boldsymbol{\theta}$ \\
\hline $\mathrm{BLB} 1_{125}$ & 6 & 4 & 0 & 0 & 2 & 1 & 2 & 5.45 & 12.26 & 0.44 \\
\hline $\mathrm{BLB2} 2_{125}$ & 7 & 3 & 0 & 0 & 1 & 0 & 1 & 4.02 & 7.76 & 0.52 \\
\hline $\mathrm{BLB}_{251}$ & 9 & 6 & 0 & 1 & 1 & 1 & 0 & 1.37 & 13.61 & 0.10 \\
\hline $\mathrm{BLB} 1 \& 2_{125}$ & 12 & 6 & 0 & 0 & 2 & 1 & 1 & 11.42 & 9.27 & 1.23 \\
\hline
\end{tabular}

$\mathrm{R}_{\mathrm{M}}, \mathrm{RDP}$, GENECONV, Maxchi, Chimaera and number of recombination breakpoints calculated GARD. Recombination rate ( $\left.\rho\right)$, mutation rate $(\theta)$ and the ratio ( $\left.\rho / \theta\right)$ were calculated using LDhat.

which are an indication for positive or balancing selection acting on the BLB2 locus. On the other hand, we found these values negative for $B L B 1_{125}$ and the combination of BLB1\&2 125 , which is generally a sign of negative or purifying selection. None of these values were significant, however, so that they have to be interpreted as indication rather than evidence. A sliding window analysis of Tajima's D along the BLB1 125 and $\mathrm{BLB}_{125}$ further disentangled this pattern of differences in selection at specific sites between the two loci (Figure 3).

We calculated the relative rates of non-synonymous $\left(d_{N}\right)$ and synonymous $\left(d_{S}\right)$ substitutions for all sites, the PBR and the non-PBR according to Tong et al. (2006) (Table 5). For the PBR, the values for the ratio $d_{N} / d_{S}$ were considerably larger than 1 in all fragments, which was significant in the case of BLB2 251 . This is considered to be evidence for positive selection acting on the PBR. Moreover, both $d_{N}$ and $d_{S}$ were significantly higher at the PBR sites compared to the non-PBR sites for all fragments (Mann-Whitney $U$-test, all $\mathrm{p}<0.01$ ), a pattern which has recently been explained to be created by gene conversion combined with positive selection [78]. Synonymous mutations hitchhike alongside beneficial nonsynonymous mutations and thereby occur more frequent than under neutral expectations.

Positive selection on specific codon sites was detected using the maximum likelihood method CODEML implemented in PAML4.6 [74]. Two pairs of models were applied: M2a versus M1a, and M8 versus M7. The

Table 4 Recombinant sequences and their potential parental sequences at the black grouse BLB1 and BLB2 (calculated with Maxchi)

\begin{tabular}{|c|c|c|c|}
\hline Locus & Recombinant sequence & $\begin{array}{l}\text { Major } \\
\text { parent }\end{array}$ & $\begin{array}{l}\text { Minor } \\
\text { parent }\end{array}$ \\
\hline \multirow[t]{2}{*}{$\mathrm{BLB1}_{125}$} & $\mathrm{BLB}_{125}-03$ & $B L B 1_{125}-06$ & $\mathrm{BLB}_{125^{-14}}$ \\
\hline & $\begin{array}{l}\mathrm{BLB1}_{125^{-0}} 04, \mathrm{BLB1}_{125^{-11}} \\
\mathrm{BLB1}_{125^{-14}}\end{array}$ & $B L B 1_{125}-06$ & $\mathrm{BLB}_{125}-05$ \\
\hline $\mathrm{BLB}_{125}$ & $\begin{array}{l}\mathrm{BLB2}_{125}-07, \mathrm{BLB2}{ }_{125}-09 \\
\mathrm{BLB2} 2_{125^{-12}}\end{array}$ & $B L B 2_{125}-04$ & unknown \\
\hline$B L B 2_{251}$ & $B L B 2_{251}-04 B$ & $B L B 2_{251}-07$ & $B L B 2_{251}-10$ \\
\hline \multirow[t]{2}{*}{ BLB1\&2 125} & $\mathrm{BLB1}_{125}-05$ & $B L B 2_{125}-07$ & $\mathrm{BLB2}{ }_{125}-02$ \\
\hline & $\begin{array}{l}\mathrm{BLB}_{125^{-07}}, \mathrm{BLB} 2_{125^{-09}} \\
\mathrm{BLB2}{ }_{125^{-12}} \mathrm{BLB1}{ }_{125^{-03}}\end{array}$ & $\mathrm{BLB} 1_{125}-06$ & $\overline{B L B 2}{ }_{125}-01$ \\
\hline
\end{tabular}

models M2a and M8, which allow for positive selection, fitted the data significantly better than the neutral models for both BLB loci (Table 6 and Additional file 4). Several codons were identified as significantly positively selected ( $\omega>1$, Table 6 and Figure 2). Four selected codons were identified with both M2a and M8 for BLB1 125 and four and five codons were identified with M2a and M8, respectively, for $B L B 22_{125}$. Codons 71,74 and 86 were identified as positively selected in both $B L B 1_{125}$ and $B L B 2_{125}$. In addition, position 67 was identified in $B L B 1_{125}$ and position 61 and 85 in $B L B 2_{125}$. The longer fragment $B L B 2_{251}$ confirmed the same positively selected codons as calculated for the fragment BLB2 ${ }_{125}$, and revealed one additional position at the $5^{\prime}$-end of the molecule (position 9). All identified positively selected codon positions were identical or directly situated next to a peptide binding site as identified by Tong et al. [73].

\section{Reconstruction of the phylogenetic relationships}

In order to take a closer look at the orthology of the black grouse BLB1 and BLB2 with the corresponding BLB loci in the chicken and pheasant, we constructed a phylogenetic tree of the BLB1 and BLB2 3'UT regions (Figure 4), which are untranslated DNA sequences containing regulatory

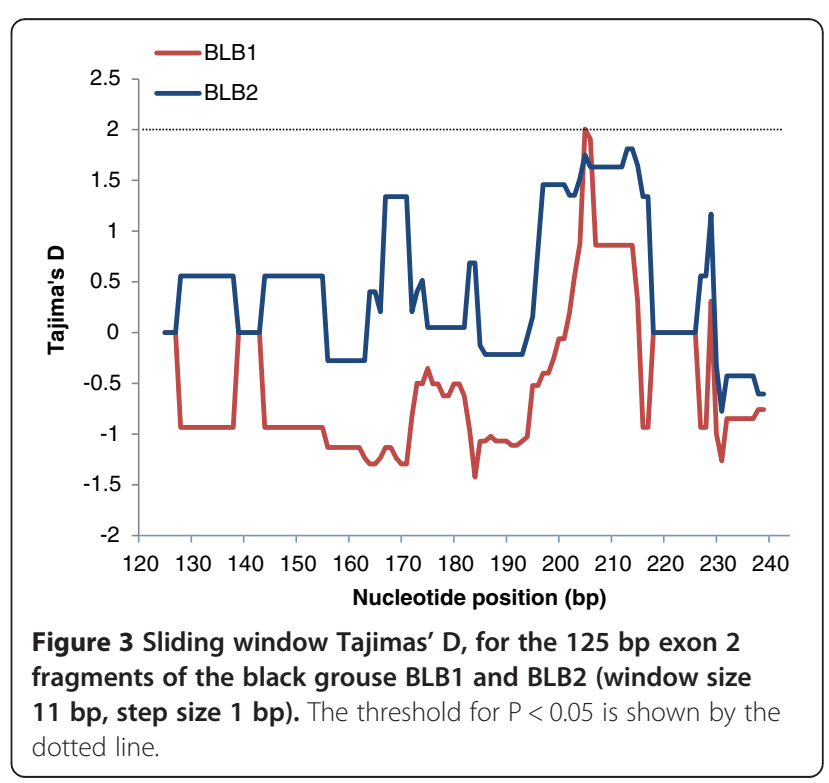


Table 5 Relative rates of non-synonymous $\left(d_{N}\right)$ and synonymous $\left(d_{s}\right)$ substitutions with standard errors calculated for the two MHC class IIB loci, averaged over all sites, the peptide binding region (PBR) and non-PBR according to Tong et al. [73]

\begin{tabular}{|c|c|c|c|c|c|c|}
\hline Positions & Locus & $d_{N} \pm S E$ & $d_{S} \pm S E$ & $d_{N} / d_{S}$ & Z & $p$ \\
\hline \multirow[t]{4}{*}{ All } & $\mathrm{BLB1}_{125}$ & $0.14 \pm 0.04$ & $0.17 \pm 0.05$ & 0.80 & -0.61 & 0.54 \\
\hline & $\mathrm{BLB}_{125}$ & $0.11 \pm 0.04$ & $0.11 \pm 0.05$ & 1.00 & 0.01 & 0.99 \\
\hline & $\mathrm{BLB2}_{251}$ & $0.11 \pm 0.04$ & $0.07 \pm 0.02$ & 1.46 & 1.06 & 0.29 \\
\hline & BLB1 \&2 125 & $0.12 \pm 0.04$ & $0.14 \pm 0.05$ & 0.88 & -0.33 & 0.74 \\
\hline \multirow[t]{4}{*}{ PBR } & $\mathrm{BLB1}_{125}$ & $0.57 \pm 0.22$ & $0.31 \pm 0.21$ & 1.84 & 1.37 & 0.17 \\
\hline & $\mathrm{BLB2}_{125}$ & $0.53 \pm 0.24$ & $0.27 \pm 0.20$ & 1.95 & 1.05 & 0.30 \\
\hline & BLB2 $251_{21}$ & $0.41 \pm 0.12$ & $0.16 \pm 0.10$ & 2.55 & 2.16 & $0.03^{*}$ \\
\hline & BLB1\&2 125 & $0.54 \pm 0.20$ & $0.29 \pm 0.20$ & 1.84 & 1.23 & 0.22 \\
\hline \multirow[t]{4}{*}{ non PBR } & $\mathrm{BLB1}_{125}$ & $0.06 \pm 0.02$ & $0.14 \pm 0.06$ & 0.42 & -1.64 & 0.10 \\
\hline & $\mathrm{BLB}_{125}$ & $0.04 \pm 0.02$ & $0.08 \pm 0.04$ & 0.56 & -0.85 & 0.41 \\
\hline & $\mathrm{BLB2}_{251}$ & $0.05 \pm 0.01$ & $0.05 \pm 0.02$ & 0.92 & -0.15 & 0.88 \\
\hline & BLB1\&2 125 & $0.05 \pm 0.02$ & $0.11 \pm 0.04$ & 0.47 & -1.58 & 0.18 \\
\hline
\end{tabular}

sequences. It has been shown that the BLB loci in both chicken and pheasant can be clearly distinguished by this part of the gene [47], in terms of nucleotide divergence and length. We found that the 3'UTR's of the three species cluster according to gene and not according to species, forming one distinct BLB1 cluster and one BLB2 cluster, and similar differences in length. This suggests orthology of the BLB1 gene between the black grouse, chicken and pheasant, and orthology of the BLB2 gene between the three species.

A contrasting phylogenetic relationship is shown in the neighbour network of the exon 2 sequences of BLB1 and BLB2 (Figure 5a). Here, sequences cluster according to species and not according to gene. All black grouse sequences cluster together, with BLB1 and BLB2 sequences intermingling. The black grouse cluster is distinct from the sequences of the domestic chicken and red jungle fowl, which form a mixed cluster, again BLB1 and BLB2 intermingling. The same picture is apparent in a neighbour network based on the third codon positions of exon 2, at which variation is mostly synonymous and therefore likely neutral. Further, exon 3 sequences of BLB1 and BLB2 repeat the same clustering according to species (see Additional file 5).

\section{Discussion}

The chicken MHC stands out with an unparalleled simple and compact architecture compared to mammals or other avian groups like the passerines [26,36]. The tight linkage of MHC genes and reduced recombination rates were suggested to have resulted in a close co-evolution of genes within MHC haplotypes ([40] but see [39]), leading to strong disease-associations of specific MHC haplotypes in consequence. In the evolutionary history of the avian MHC it is still not resolved whether this unique MHC structure in the chicken is a domestication artefact or was present before domestication as a galliform feature.

We here present a study on diversity, selection patterns and the co-evolutionary history of two transcribed MHC class IIB genes in a wild galliform bird species, the black grouse. We found evidence for both intra- and interlocus recombination or gene conversion, as well as indication for positive selection on the PBR at both loci. However, we also detected differences in the selection between the two loci, as positive selection was indicated at the BLB2 locus and purifying selection at the BLB1 locus. We were able to relate our findings to the

Table 6 Summary table for CODEML

\begin{tabular}{|c|c|c|c|c|c|c|c|c|c|}
\hline \multirow[t]{2}{*}{ Locus } & \multirow[t]{2}{*}{ Model (ref) } & \multirow[t]{2}{*}{$2\left(L_{b}-L_{a}\right)$} & \multirow[t]{2}{*}{$p$} & \multicolumn{6}{|c|}{ Positively selected codons } \\
\hline & & & & 9 & 61 & 71 & 74 & 85 & 86 \\
\hline \multirow[t]{2}{*}{$\mathrm{BLB1}_{125}$} & M1a- M2a & 34.48 & $<0.001$ & & $x$ & $\mathbf{x}$ & $x$ & & $x$ \\
\hline & M7 - M8 & 35.56 & $<0.001$ & & $x$ & $\mathbf{x}$ & $\mathbf{x}$ & & $x$ \\
\hline \multirow[t]{2}{*}{$\mathrm{BLB2}_{125}$} & M1a-M2a & 22.90 & $<0.001$ & & $x$ & $x$ & $x$ & & $x$ \\
\hline & M7 - M8 & 23.02 & $<0.001$ & & $x$ & $x$ & $x$ & $\mathrm{x}$ & $x$ \\
\hline \multirow[t]{2}{*}{$\mathrm{BLB}_{251}$} & M1a-M2a & 27.08 & $<0.001$ & & $x$ & $x$ & $x$ & & $x$ \\
\hline & M7 - M8 & 27.12 & $<0.001$ & $x$ & $x$ & $x$ & $x$ & $x$ & $\mathbf{x}$ \\
\hline \multirow[t]{3}{*}{$B L B 1 \& 2_{125}$} & M1a-M2a & 45.40 & $<0.001$ & & $x$ & $x$ & $x$ & $x$ & $x$ \\
\hline & M7 - M8 & 48.38 & $<0.001$ & & $x$ & $\mathrm{x}$ & $x$ & $x$ & $x$ \\
\hline & Dist. to Tong PBR & & & 0 & 1 & 0 & 0 & 1 & 0 \\
\hline
\end{tabular}

Two model comparisons were conducted; each comparison includes a neutral model (M1a, M7) and a model allowing for positive selection (M2a, M8). Significance was assessed by comparing twice the difference in likelihood $2\left(L_{b}-L_{a}\right)$ between the models to a $x^{2}$-distribution (df $=2$ ). The models fitting our data best are marked in bold. $x$ marks positively selected codons calculated by Bayes Empirical Bayes (BEB) at the $>95 \%$ confidence level. Distance (Dist.) to Tong PBR corresponds to the amino acid distance between identified site and the nearest peptide binding position identified by Tong et al. [73]. For parameter estimates see Additional file 4. 


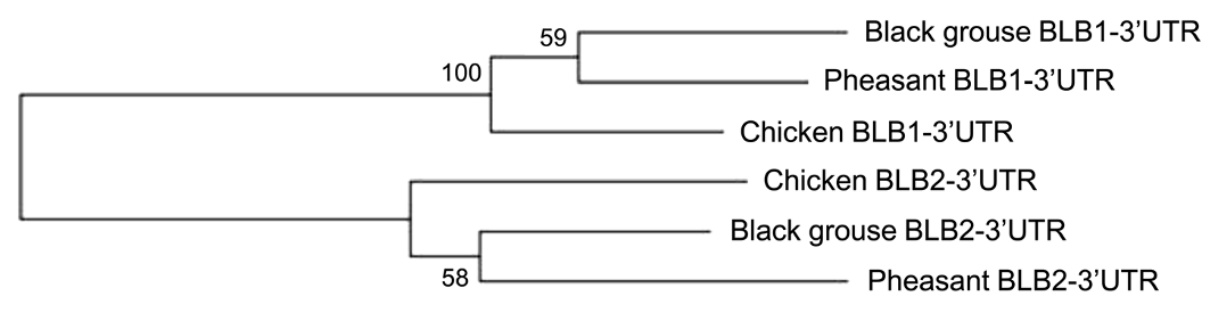

0.05

Figure 4 Neighbour joining tree for the 3'UTR. BLB1 and BLB2 sequences derived from black grouse (fosmid individual JHGO 213 [48], chicken [GenBank AB268588] and pheasant [GenBank AJ224349]. The scale bar represents substitutions per site.

structure of the chicken MHC class II by resolving orthology to the chicken BLB loci.

Similar to the work of [22] on red jungle fowl, we amplified the two black grouse BLB loci separately in twelve individuals. Eleven of the individuals included in this study were cloned in our previous studies with non locus-specific primers that gave a 125 bp BLB exon 2 product $[15,50]$. The resulting sequences showed that individuals carried between two and four BLB alleles, without locus assignment. This is a slight underestimation in number of alleles per individual compared to the here presented study, which revealed between three and four alleles per individual for BLB1 and BLB2 combined. Part of this additional variation was detected through the longer BLB2 fragment, which revealed further variable positions towards the $5^{\prime}$-end of the exon 2. To another part, the underestimation by the cloning/sequencing approach may be a result of too few analysed clones $(16-26$ per individual). On the other hand, for individual D248 the cloning procedure revealed one allele $\left(\mathrm{BLB}^{*}{ }_{125}-04\right)$ which could not be confirmed by the single-locus amplification. This additional allele is likely to be a contamination during the cloning process. Considering that cloning is more time-consuming than direct sequencing, can add artefacts derived from mismatch repair or the formation of chimeric sequences [53] and is more contamination-prone, the here presented single locus sequence-based typing method clearly outmatches the cloning/sequencing approach in both accuracy and effort.

Another finding in this study concerns the expression of the two BLB loci in black grouse. We earlier amplified BLB cDNA sequences for individual D870 [15], which we could show now derived from both BLB1 and BLB2. Thus, both BLB1 and BLB2 are transcribed loci in black grouse. In domestic chicken BLB2 is considered to be dominantly expressed [44,45], while BLB1 is less expressed and has even been suggested to be neutral to selection and not involved in peptide binding $[30,46]$. This ultimately leads to the question whether the BLB1 and BLB2 loci in black grouse show a similar situation or differ in the degree and mode of selection compared to the chicken. We therefore compared diversity and selection patterns between BLB1 and BLB2 to explore their respective modes of selection.

We observed significant linkage between BLB1 and BLB2, in line with the hypothesis of Kaufman [30,40] that MHC genes in chicken form a tightly linked cluster of co-evolving genes. Similarly, Agudo et al. [79] showed in a study on Egyptian vultures (Neophron percnopterus) the existence of linkage groups, containing pairs of $\mathrm{MHC}$ alleles in strong linkage disequilibrium. They interpret the presence of linkage groups with similar MHC alleles as indication of concerted evolution acting on the MHC gene duplicates. However, concerted evolution does not necessarily homogenize a gene cluster. Gene conversion can both homogenize and diversify among paralogues, as was shown for the gene family $h s p 70$ in Drosophila [80].

Intuitively one might think that gene conversion and recombination should tend to break up linkage disequilibrium. But this does not seem to be this simple. The human olfactory receptor (OR) gene cluster is another gene family suggested to evolve under concerted evolution with gene conversion events [81]. Linkage disequilibrium is highly significant in the centromeric part of the gene cluster, whereas no linkage disequilibrium could be observed in the telomeric part of the cluster. These considerable differences suggest the presence of several recombination hotspots within the OR cluster.

In terms of number of different alleles, MHC diversity was similar at the two BLB loci, however, BLB2 revealed higher heterozygosity and was less variable in terms of nucleotide diversity than BLB1. Signals of positive selection were found on both the BLB1 and BLB2 locus. Elevated $d N / d S$ ratios were obtained at the PBR of both loci, though only significant for the longer BLB2 fragment. Maximum likelihood analyses confirmed that models allowing for positive selection fitted our data significantly better than neutral models. With these models, four identical codons were identified to be under significant positive selection in BLB1 and BLB2, and two additional positions were identified for the BLB2 locus. All 

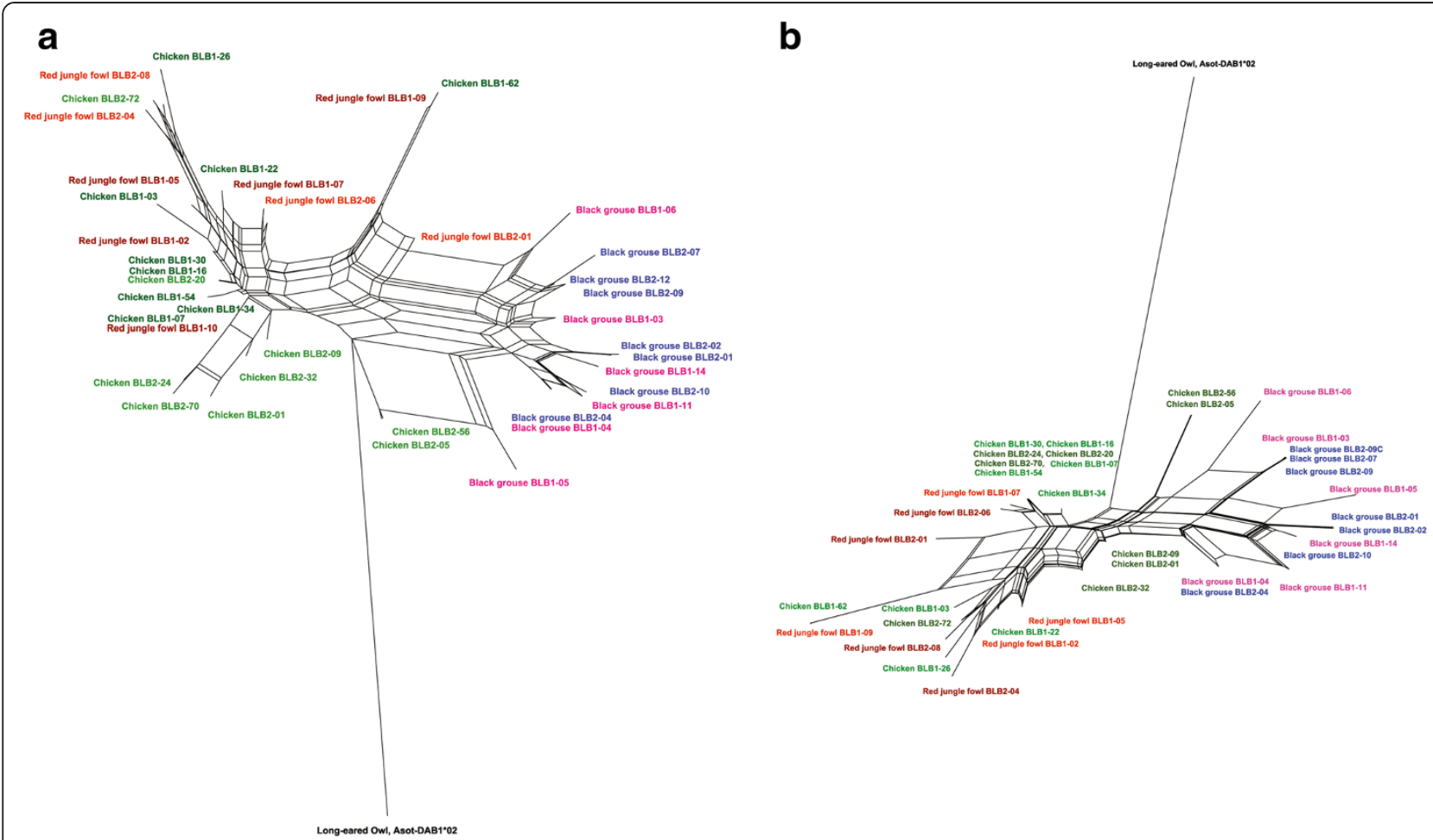

Figure 5 Neighbour network for BLB1 and BLB2. Sequences of the black grouse (pink and blue), chicken (dark and light green) [GenBank AB426144, AB426150-51, AJ248577, M29763] and red jungle fowl (brown and orange) [GenBank AM489767 - AM489776] for the a) 125 nucleotide sequence of exon 2 and $\mathbf{b}$ ) only $3^{\text {rd }}$ codon positions in the 125 nucleotide sequence of exon 2. Long-eared Owl Asot-DAB1*02 [GenBank EF641225] was used as an outgroup.

of these positively selected codon positions were either congruent or directly adjacent to a peptide binding site as identified by Tong et al. [73] for human MHC class II molecules. This congruence emphasises the structural similarity of codon positions involved in peptide binding throughout evolutionary lineages. However, it has been suggested the methods used here might be prone to overestimate positive selection, particularly when recombination rate is high [82].

A different pattern of selection was found, however, regarding neutrality tests as Tajima's D. We found a negative Tajima's D value for BLB1, indicative of purifying selection, and a positive Tajima's D for BLB2 indicating positive or balancing selection, although not significant in both cases. In a sliding window analysis of Tajima's D comparing BLB1 and BLB2 it became more apparent that these different selection footprints are due to differences at some specific nucleotide sites only, while at other positions positive selection between the two loci coincides. These opposite but vague signals of differential selection at BLB1 and BLB2 made us explore this further. Worley et al. [22] found likewise a negative Tajima's D for BLB1 and positive value for BLB2 (both n.s.) in a captive population of red jungle fowl but the authors points out that a negative Tajima's D could also be a result of a population bottleneck. As a further comparison, we obtained domestic chicken BLB1 and BLB2 sequences from GenBank and calculated Tajima's D the same way as we did for the black grouse sequences. This analysis repeated the observation of positive Tajima's D for BLB2 and negative for BLB1 (data not shown). We also observed that theta $k$ and $\pi$ were higher in BLB1 than BLB2 in the domestic chicken, which was likewise observed for black grouse. In conclusion, we interpret this as a repeated pattern of differential selection on BLB1 and BLB2 in galliform birds.

Detecting recombination or gene conversion events using statistical methods can be highly problematic, in particular when small fragments are transferred or the gene conversion rate is too high. Hence, we have to keep in mind that a lack of evidence is no evidence for absence. To minimize the risk of missing the footprint of gene conversion, it is recommended to use multiple statistical methods for detection [58]. Following this recommendation we applied a set of seven statistical methods, which have been evaluated to be powerful and accurate for different conditions and scenarios [59]. While two of the methods (RDP and GENECONV) failed to detect most recombination signals, the other methods detected recombination or gene conversion both within and 
'between the two BLB loci. As an additional hint for the occurrence of gene conversion, we detected significantly higher $d_{N}$ and $d_{S}$ values at the PBR sites compared to the non-PBR sites at both loci. This situation is likely to be created by a combination of positive selection and gene conversion [78,83]. Gene conversion events transferring advantageous non-synonymous substitutions at the PBR are positively selected, and in doing so synonymous substitutions are carried along. This way, synonymous substitutions appear more often at the PBR than expected by point mutations under neutrality. We conclude that both intra- and interlocus genetic exchange play an important role in shaping the black grouse MHC class II.

The 3'UTRs between BLB1 versus BLB2 differ in domestic chicken as well as between the corresponding DAB1 and DAB2 loci in pheasants in both length and nucleotide composition, so that the sequences cluster together as orthologous genes rather than according to species [47]. We have observed similar differences in the 3'UTRs between black grouse BLB1 and BLB2 and could prove orthology of the BLB1 gene between the black grouse, chicken and pheasant, and orthology of the BLB2 gene between the three species. The origin of the pheasant like birds Phasianoidea is estimated to approximately 40 million years ago [84] and chicken and black grouse/turkey diverged approximately 30 million years ago [85]. It seems that the duplication of BLB1 and BLB2 is a case of pre-speciation duplication that has arisen in the ancestral species before the split into chicken, pheasant and black grouse. The observed differences in the length of the 3'UTRs between the two loci may also reflect a difference in function [86].

In all other phylogenetic reconstructions based on the exon 2 , the third codon positions of exon 2 and the exon 3 , sequences clustered species-specific and not locusspecific. This is indication for frequent interlocus genetic exchange homogenizing sequences between the paralogous loci, as suggested by Wittzell et al. [47]. The fact that the whole exon 2 sequences and third codon positions of the exon 2 networks present similar phylogenetic relations contains more indication for an early duplication in the evolutionary history of BLB1 and BLB2. Under convergent selection on the two loci, the two phylogenies would reveal deviating patterns [34]. A phylogenetic tree based on the codon sequences should reflect functional similarities, whereas the third codon positions are expected to mirror the neutral gene history. Under the early duplication hypothesis, in contrast, a phylogeny based on the third codon positions will match the phylogenetic relation based on the whole codon sequences, as in our case. Note that in this analysis all third codon positions are considered and not only the synonymous positions at the PBR, which are possibly under a selective sweep of the positions under selection, as discussed earlier.
In summary, we infer that the BLB gene duplicated before the species divergence into chicken, black grouse and pheasant and thus is a case of pre speciation duplication. Further, we conclude that BLB1 and BLB2 in black grouse are subjected to homogenizing concerted evolution due to inter-genetic exchange between loci after species divergence. Different selection patterns indicated for BLB1 and BLB2 may be a sign of different immunogenetic functions. Both BLB1 and BLB2 have been under balancing selection during their evolutionary history. Nevertheless, it is likewise possible that, at present, balancing selection may be operating directly only on BLB2. Because of the tight linkage between the loci, BLB1 would be hitchhiking with BLB2.

\section{Conclusions}

We have presented a powerful single locus genotyping method for amplifying MHC class IIB loci in black grouse. This method will allow exploring correlations between MHC heterozygosity and reproductive success, mate choice and disease resistance in this and related species.

It is evident that both BLB1 and BLB2 are expressed and under balancing selection in black grouse. Even though we found some differences between the loci in selection patterns, the similarities between loci were apparent. In fact, our data show inter-locus genetic exchange between BLB1 and BLB2. The loci are in linkage disequilibrium, which is in line with what have been stated regarding tightly coevolving genes within $\mathrm{MHC}$ under the minimal essential MHC hypothesis [31,36]. Our results support the conclusion that $\mathrm{MHC}$ form and function in birds derived from studies on the domesticated chicken are not artefacts of the domestication process. However, the data from black grouse do suggest more recombination than previously observed in chicken (but see [39]). These data are important for understanding how the MHC of birds and other non-mammalian vertebrates have evolved.

The duplicated genes BLB1 and BLB2 are a case of early duplication and have co-evolved in a concerted way by interlocus gene exchange not only in chicken but also in black grouse. This supports the so far nontested hypothesis of concerted evolution of BLB1 and BLB2 in galliformes [47].

\section{Additional files}

Additional file 1: MHC primers and corresponding amplification

lengths. The fragment column highlights the locus and region amplified with each primer pair. The PCR product length is given without primers.

Additional file 2: Best evolutionary models estimated by Aikaike's information criterion in jModelTest. The models were used to construct phylogenetic trees. 
Additional file 3: Alignment of black grouse MHC class II B exon 2 nucleotide sequences. Locus-designated sequences derived in the present study are indicated with $125 \mathrm{bp}$ and $251 \mathrm{bp}$. Sequences indicated as $\mathrm{BLB}^{*}$ are cloned in previous studies and not designated to locus, for example 202 bp cDNA sequences (from individual D870, see Table 1). Sequences likely to be from the same allele are grouped by grey shading.

Additional file 4: Supplement for the test for positive selection.

Likelihood values and parameter estimates for the different models calculated with CODEML implemented in PAML 4.6 [74]. M = model, InL = Log-likelihood value. In the neutral model $\mathrm{MO}, \omega$ is equivalent to averaged $d N / d S$. Dark grey shade highlights the significantly best models.

Additional file 5: Neighbour Joining tree for exon 3. BLB1 and BLB2 sequences derived from black grouse (fosmid individual JHGO 213 [48] [GenBank JQ028669] and chicken [GenBank AB268588]. Goose [GenBank EU999169] was used as an outgroup.

\section{Competing interests}

The authors declare that they have no competing interests.

\section{Authors' contributions}

TS and JH conceived and designed the study, TS and BW carried out the molecular genetic studies. TS, YML and BW performed the statistical analyses and interpretation of the data. The manuscript was written by TS and YML, with input from $\mathrm{JH}$ and BW. All authors read and approved the final manuscript.

\section{Acknowledgements}

Thanks to Heli Siitari, Rauno Alatalo, and Sten Svartaas for providing black grouse samples. We are grateful to Gunilla Engström for assistance in the lab, to Robert Ekblom and three anonymous reviewers for valuable comments on the manuscript and Reto Burri for helpful discussions. Thanks also to Eleanor Jones and Radoslav Kozma for language improvements. This project was funded through the Swedish Research Council (VR) to JH.

\section{Author details}

'Population Biology and Conservation Biology, Department of Ecology and Genetics, Evolutionary Biology Center, Uppsala University, Norbyvägen 18D, SE-752 36, Uppsala, Sweden. ${ }^{2}$ Current address: Swedish Institute for Communicable Disease Control, Department of Analysis and Prevention, Nobels väg 18, SE-171 82, Solna, Sweden.

Received: 24 October 2012 Accepted: 12 April 2013

Published: 24 April 2013

\section{References}

1. Lynch M, Conery JS: The evolutionary fate and consequences of duplicate genes. Science 2000, 290(5494):1151-1155.

2. Klein J: Natural history of the major histocompatibility complex. New York: John Wiley and sons; 1986.

3. Spurgin LG, Richardson DS: How pathogens drive genetic diversity: MHC mechanisms and misunderstandings. Proc R Soc B-Biolo Scie 2010, 277(1684):979-988

4. Piertney S, Oliver M: The evolutionary ecology of the major histocompatibility complex. Heredity 2006, 96(1):7-21.

5. Robinson J, Mistry K, McWilliam H, Lopez R, Parham P, Marsh SGE: The IMGT/HLA database. Nucleic Acids Res 2011, 39(suppl 1):D1171-D1176.

6. Apanius $V$, Penn D, Slev PR, Ruff $L R$, Potts WK: The nature of selection on the major histocompatibility complex. Crit Rev Immunol 1997, 17(2):179-224.

7. Bernatchez $L$, Landry C: MHC studies in nonmodel vertebrates: what have we learned about natural selection in 15 years? J Evolution Biol 2003, 16(3):363-377.

8. Hughes AL, Yeager M: Natural selection at major histocompatibility complex loci of vertebrates. Annu Rev Genet 1998, 32:415-435.

9. Martinsohn JT, Sousa AB, Guethlein LA, Howard JC: The gene conversion hypothesis of MHC evolution: a review. Immunogenetics 1999, 50(3):168-200.

10. Klein J: Origin of major histocompatibility complex polymorphism: the transspecies hypothesis. Hum Immunol 1987, 19:155-162.

11. van Oosterhout C: A new theory of MHC evolution: beyond selection on the immune genes. Proc R Soc B-Biol Scie 2009, 276(1657):657-665.
12. Edwards SV, Gasper J, March M: Genomics and polymorphism of AgphDAB1, an MHC class II B gene in red-winged blackbirds (Agelaius phoeniceus). Mol Biol Evol 1998, 15(3):236-250.

13. Ekblom R, Grahn M, Höglund J: Patterns of polymorphism in the MHC class II of a non-passerine bird, the great snipe (Gallinago media). Immunogenetics 2003, 54(10):734-741.

14. Miller HC, Allendorf F, Daugherty $\mathrm{CH}$ : Genetic diversity and differentiation at MHC genes in island populations of tuatara (Sphenodon spp.). Mol Ecol 2010, 19(18):3894-3908.

15. Strand T, Westerdahl H, Höglund J, Alatalo RV, Siitari H: The MHC class II of the Black grouse (Tetrao tetrix) consists of low numbers of $B$ and $Y$ genes with variable diversity and expression. Immunogenetics 2007, 59(9):725-734.

16. Westerdahl $H$, Wittzell $H$, von Schantz T: Polymorphism and transcription of Mhc class I genes in a passerine bird, the great reed warbler. Immunogenetics 1999, 49(3):158-170.

17. Miller HC, Lambert DM: Genetic drift outweighs balancing selection in shaping post-bottleneck major histocompatibility complex variation in New Zealand robins (Petroicidae). Mol Ecol 2004, 13(12):3709-3721.

18. Hughes $C R$, Miles $S$, Walbroehl JM: Support for the minimal essential MHC hypothesis: a parrot with a single, highly polymorphic MHC class IIB gene. Immunogenetics 2008, 60(5):219-231.

19. Tsuda $T$, Tsuda M, Naruse T, Kawata H, Ando A, Shiina T, Fukuda M, Kurita M, LeMaho I, Kulski JK, et al: Phylogenetic analysis of penguin (Spheniscidae) species based on sequence variation in MHC class II genes. Immunogenetics 2001, 53(8):712-716.

20. Alcaide M, Edwards S, Negro J: Characterization, polymorphism, and evolution of MHC class II B genes in birds of prey. J Mol Evol 2007, 65:541-554.

21. O'Neill A, Livant E, Ewald S: The chicken <i > BF1 (classical MHC class I) gene shows evidence of selection for diversity in expression and in promoter and signal peptide regions. Immunogenetics 2009, 61(4):289-302.

22. Worley K, Gillingham M, Jensen P, Kennedy L, Pizzari T, Kaufman J, Richardson DS: Single locus typing of MHC class I and class IIB loci in a population of red jungle fowl. Immunogenetics 2008, 60(5):233-247.

23. Reed K, Bauer M, Monson M, Benoit B, Chaves L, O'Hare T, Delany M: Defining the Turkey MHC: identification of expressed class I- and class IIB-like genes independent of the MHC-B. Immunogenetics 2011, 63:753-771.

24. Burri R, Niculita-Hirzel H, Roulin A, Fumagalli L: Isolation and characterization of major histocompatibility complex (MHC) class II B genes in the Barn owl (Aves: Tyto alba). Immunogenetics 2008, 60(9):543-550.

25. Kelley J, Walter L, Trowsdale J: Comparative genomics of major histocompatibility complexes. Immunogenetics 2005, 56(10):683-695.

26. Hess CM, Edwards SV: The Evolution of the Major Histocompatibility Complex in Birds. Bioscience 2002, 52(5):423-431.

27. Kulski J, Shiina T, Anzai T, Kohara S, Inoko H: Comparative genomic analysis of the MHC: the evolution of class I duplication blocks, diversity and complexity from shark to man. Immunol Rev 2002, 190(1):95-122.

28. Nei M, Gu X, Sitnikova T: Evolution by the birth-and-death process in multigene families of the vertebrate immune system. Colloquium on Genetics and the Origin of Species: Jan 30-Feb 01 1997; Irvine, Ca: Natl Acad Sciences 1997, 94:7799-7806.

29. Nei M, Rooney A: Concerted and birth-and-death evolution of multigene families. Ann Rev Gen 2005, 39:121-152.

30. Kaufman J: The avian MHC. In Avian Immunology. Edited by Davison F, Kaspers B, Schat KA. Oxford: Academic; 2008.

31. Walker BA, Hunt LG, Sowa AK, Skjødt K, Göbel TW, Lehner PJ, Kaufman J: The dominantly expressed class I molecule of the chicken MHC is explained by coevolution with the polymorphic peptide transporter (TAP) genes. Proc Natl Acad Sci 2011, 108(20):8396-8401.

32. Burri R, Hirzel H, Salamin N, Roulin A, Fumagalli L: Evolutionary patterns of MHC class IIB in owls and their implications for the understanding of avian MHC evolution. Mol Biol Evol 2008, 25(6):1180-1191.

33. Fawcett J, Innan H: Neutral and Non-Neutral Evolution of Duplicated Genes with Gene Conversion. Genes 2011, 2(1):191-209.

34. Burri R, Salamin N, Studer RA, Roulin A, Fumagalli L: Adaptive divergence of ancient gene duplicates in the Avian MHC Class II $\beta$. Mol Biol Evol 2010, 27(10):2360-2374.

35. Briles WE, McGibbon WH, Irwin MR: On multiple alleles effecting cellular antigens in the chicken. Genetics 1950, 35(6):633-652.

36. Kaufman J, Milne S, Gobel TWF, Walker BA, Jacob JP, Auffray C, Zoorob R, Beck S: The chicken B locus is a minimal essential major histocompatibility complex. Nature 1999, 401(6756):923-925. 
37. The MHC sequencing consortium: Complete sequence and gene map of a human major histocompatibility complex. Nature 1999, 401(6756):921.

38. Balakrishnan C, Ekblom R, Volker M, Westerdahl H, Godinez R, Kotkiewicz H, Burt D, Graves T, Griffin D, Warren W, et al: Gene duplication and fragmentation in the zebra finch major histocompatibility complex. BMC Bio/ 2010, 8(1):29.

39. Hosomichi K, Miller MM, Goto RM, Wang YJ, Suzuki S, Kulski JK, Nishibori M, Inoko H, Hanzawa K, Shiina T: Contribution of mutation, recombination, and gene conversion to chicken MHC-B haplotype diversity. J Immunol 2008, 181(5):3393-3399.

40. Kaufman J: Co-evolving genes in MHC haplotypes: the "rule" for nonmammalian vertebrates? Immunogenetics 1999, 50(3-4):228-236

41. Skjodt $\mathrm{K}$, Koch C, Crone M, Simonsen M: Analysis of chickens for recombination within the MHC (B-complex). Tissue Antigens 1985, 25(5):278-282.

42. Chaves LD, Faile GM, Krueth SB, Hendrickson JA, Reed KM: Haplotype variation, recombination, and gene conversion within the turkey MHC-B locus. Immunogenetics 2010, 62(7):465-477.

43. Shiina T, Shimizu S, Hosomichi K, Kohara S, Watanabe S, Hanzawa K, Beck S, Kulski JK, Inoko H: Comparative genomic analysis of two avian (quail and chicken) MHC regions. J Immunol 2004, 172(11):6751-6763.

44. Jacob JP, Milne $S$, Beck S, Kaufman J: The major and a minor class II beta-chain (B-LB) gene flank the Tapasin gene in the B-F/B-L region of the chicken major histocompatibility complex. Immunogenetics 2000

51(2):138-147.

45. Pharr GT, Dodgson JB, Hunt HD, Bacon LD: Class II MHC cDNAs in 15I(5) B-congenic chickens. Immunogenetics 1998, 47(5):350-354.

46. Shaw I, Powell TJ, Marston DA, Baker K, van Hateren A, Riegert P, Wiles MV, Milne S, Beck S, Kaufman J: Different evolutionary histories of the two classical class I genes BF1 and BF2 illustrate drift and selection within the stable MHC haplotypes of chickens. J Immunol 2007 , 178(9):5744-5752.

47. Wittzell $H$, Madsen $T$, Westerdahl $H$, Shine $R$, von Schantz $T$ : MHC variation in birds and reptiles. Genetica 1999, 104(3):301-309.

48. Wang B, Ekblom R, Strand T, Portela-Bens S, Hoglund J: Sequencing of the core $\mathrm{MHC}$ region of black grouse (Tetrao tetrix) and comparative genomics of the galliform MHC. BMC Genomics 2012, 13(1):553.

49. Li XX, Han LX, Han JL: No Specific Primer Can Independently Amplify the Complete Exon 2 of Chicken BLB1 or BLB2 Genes. Int J Poultry Scie 2010, 9(2):192-197.

50. Strand T, Höglund J: Genotyping of black grouse MHC class II B using reference Strand-Mediated Conformational Analysis (RSCA). BMC Res Notes 2011, 4(1):183.

51. Paxton RJ, Thoren PA, Tengo J, Estoup A, Pamilo P: Mating structure and nestmate relatedness in a communal bee, Andrena jacobi (Hymenoptera, Andrenidae), using microsatellites. Mol Ecol 1996, 5(4):511-519.

52. Rozen S, Skaletsky H: In Primer3 on the WWW for General Users and for Biologist Programmers Bioinformatics Methods and Protocols, Volume 132 Edited by Misener S, Krawetz SA. Humana Press; 2000:365-386.

53. Lenz $T L$, Becker $S$ : Simple approach to reduce $P C R$ artefact formation leads to reliable genotyping of $\mathrm{MHC}$ and other highly polymorphic loci Implications for evolutionary analysis. Gene 2008, 427(1-2):117-123

54. Westerdahl H, Wittzell $H$, Schantz T, Bensch S: MHC class I typing in a songbird with numerous loci and high polymorphism using motifspecific PCR and DGGE. Heredity 2004, 92(6):534-542.

55. Librado P, Rozas J: DnaSP v5: a software for comprehensive analysis of DNA polymorphism data. Bioinformatics 2009, 25:1451-1452.

56. Alcaide M, Rodriguez A, Negro J: Sampling strategies for accurate computational inferences of gametic phase across highly polymorphic major histocompatibility complex loci. BMC Res Notes 2011, 4(1):151.

57. Bos D, Turner S, Dewoody J: Haplotype inference from diploid sequence data: evaluating performance using non-neutral $\mathrm{MHC}$ sequences. Hereditas 2007, 144:228-234.

58. Mansai SP, Innan $H$ : The power of the methods for detecting interlocus gene conversion. Genetics 2010, 184(2):517-527.

59. Martin DP, Lemey P, Posada D: Analysing recombination in nucleotide sequences. Mol Ecol Resour 2011, 11(6):943-955.
60. Hudson RR, Kaplan NL: Statistical properties of the number of recombination events in the history of a sample of DNA-sequences. Genetics 1985, 111(1):147-164.

61. Martin DP, Lemey P, Lott M, Moulton V, Posada D, Lefeuvre P: RDP3: a flexible and fast computer program for analyzing recombination. Bioinformatics 2010, 26(19):2462-2463.

62. Martin D, Rybicki E: RDP: detection of recombination amongst aligned sequences. Bioinformatics 2000, 16(6):562-563.

63. Maynard Smith J: Analyzing the mosaic structure of genes. J Mol Evol 1992, 34(2):126-129.

64. Posada D, Crandall KA: Evaluation of methods for detecting recombination from DNA sequences: Computer simulations. Proc Natl Acad Sci USA 2001, 98(24):13757-13762.

65. Padidam M, Sawyer S, Fauquet CM: Possible emergence of new geminiviruses by frequent recombination. Virology 1999, 265(2):218-225.

66. Kosakovsky Pond SL, Posada D, Gravenor MB, Woelk CH, Frost SDW: Automated phylogenetic detection of recombination using a genetic algorithm. Mol Biol Evol 2006, 23(10):1891-1901.

67. Delport W, Poon AFY, Frost SDW, Pond SLK: Datamonkey 2010: a suite of phylogenetic analysis tools for evolutionary biology. Bioinformatics 2010 26(19):2455-2457.

68. McVean GAT, Myers SR, Hunt S, Deloukas P, Bentley DR, Donnelly P: The fine-scale structure of recombination rate variation in the human genome. Science 2004, 304(5670):581-584

69. Excoffier $L$, Lischer HEL: Arlequin suite ver 3.5: a new series of programs to perform population genetics analyses under Linux and Windows. Mol Ecol Resour 2010, 10(3):564-567.

70. Hurst LD: Genetics and the understanding of selection. Nat Rev Genet 2009, 10(2):83-93.

71. Garrigan D, Hedrick PW, Mitton J: Perspective: Detecting adaptive molecular polymorphism: Lessons from the MHC. Evolution 2003, 57(8):1707-1722

72. Tamura K, Peterson D, Peterson N, Stecher G, Nei M, Kumar S: MEGA5: Molecular Evolutionary Genetics Analysis Using Maximum Likelihood, Evolutionary Distance, and Maximum Parsimony Methods. Mol Biol Evol 2011 28:2731-2739.

73. Tong J, Bramson J, Kanduc D, Chow S, Sinha A, Ranganathan S: Modeling the bound conformation of Pemphigus Vulgaris-associated peptides to MHC Class II DR and DO Alleles. Immunome Res 2006, 2(1):1.

74. Yang ZH: PAML 4: Phylogenetic analysis by maximum likelihood. Mol Biol Evol 2007, 24(8):1586-1591.

75. Guindon S, Dufayard JF, Lefort V, Anisimova M, Hordijk W, Gascuel O: New algorithms and methods to estimate maximum-likelihood phylogenies: assessing the performance of PhyML 3.0. Syst Biol 2010, 59(3):307-321.

76. Posada D: jModelTest: Phylogenetic model averaging. Mol Biol Evol 2008 25(7):1253-1256.

77. Huson DH, Bryant D: Application of phylogenetic networks in evolutionary studies. Mol Biol Evol 2006, 23(2):254-267.

78. Spurgin LG, van Oosterhout C, Illera JC, Bridgett S, Gharbi K, Emerson BC, Richardson DS: Gene conversion rapidly generates major histocompatibility complex diversity in recently founded bird populations. Mol Ecol 2011, 20(24):5213-5225

79. Agudo R, Alcaide M, Rico C, Lemus JA, Blanco G, Hiraldo F, DonÁZar JA: Major histocompatibility complex variation in insular populations of the Egyptian vulture: inferences about the roles of genetic drift and selection. Mol Ecol 2011, 20(11):2329-2340.

80. Bettencourt B, Feder M: Rapid Concerted Evolution via Gene Conversion at the Drosophila hso70 Genes. J Mol Evol 2002, 54:569-586.

81. Sharon D, Gilad Y, Glusman G, Khen M, Lancet D, Kalush F: Identification and characterization of coding single-nucleotide polymorphisms within a human olfactory receptor gene cluster. Gene 2000, 260(1-2):87-94.

82. Anisimova M, Nielsen $R$, Yang Z: Effect of recombination on the accuracy of the likelihood method for detecting positive selection at amino acid sites. Genetics 2003, 164(3):1229-1236.

83. Ohta T: Gene conversion vs point mutation in generating variability at the antigen recognition site of Major Histocompatibility Complex loci. $J$ Mol Evol 1995, 41(2):115-119. 
84. Helm-Bychowski KM, Wilson AC: Rates of nuclear DNA evolution in pheasant-like birds: evidence from restriction maps. Proc Natl Acad Sci 1986, 83(3):688-692.

85. Pereira SL, Baker AJ: A molecular timescale for galliform birds accounting for uncertainty in time estimates and heterogeneity of rates of DNA substitutions across lineages and sites. Mol Phylogenet Evol 2006, 38(2):499-509.

86. Stark A, Brennecke J, Bushati N, Russell RB, Cohen SM: Animal MicroRNAs Confer Robustness to Gene Expression and Have a Significant Impact on 3'UTR Evolution. Cell 2005, 123(6):1133-1146.

doi:10.1186/1471-2156-14-29

Cite this article as: Strand et al:: Evolutionary history of black grouse major histocompatibility complex class IIB genes revealed through single locus sequence-based genotyping. BMC Genetics 2013 14:29.

\section{Submit your next manuscript to BioMed Central and take full advantage of:}

- Convenient online submission

- Thorough peer review

- No space constraints or color figure charges

- Immediate publication on acceptance

- Inclusion in PubMed, CAS, Scopus and Google Scholar

- Research which is freely available for redistribution 